\title{
Search for RS Gravitons Decaying into a Jet plus Missing $E_{\mathbf{T}}$ with CMS
}

\author{
Thiago R. F. P. Tomei* \\ On behalf of the CMS Collaboration \\ Instituto de Física Teórica, Universidade Estadual Paulista \\ E-mail: Thiago.Tomei@cern.ch
}

\begin{abstract}
We search for the production of heavy resonances in proton-proton collisions at $\operatorname{sqrt}(\mathrm{s})=7 \mathrm{TeV}$, with the Randall-Sundrum graviton $\mathrm{G}_{K K}$ as a benchmark model. We focus on the $\mathrm{G}_{K K} \rightarrow \mathrm{ZZ} \rightarrow$ $\mathrm{q} \overline{\mathrm{q}} v \bar{v}$ reaction with boosted $\mathrm{Z}$ bosons. We look for the jet plus missing transverse energy signature in the $4.7 \mathrm{fb}^{-1}$ of data collected by the CMS detector during 2011. Since the event yield is compatible with what would be expected solely from Standard Model processes, we are able to derive limits on the cross-section for the RS graviton production, and hence on the parameters of the Warped Extra Dimensions model. The cross-section 95\% confidence upper limits are found to be in the range [0.047, 0.021] pb for resonance masses ranging between 1000 and $1500 \mathrm{GeV}$. We extend the $k / M_{P l}$ search range to values up to 0.3 , and translate the cross-section limits to the $\left(M_{G}, k / M_{P l}\right)$ parameter space. In that way, we set $95 \%$ confidence upper limits on the coupling parameter $k / M_{P l}$ in the range 0.11 to 0.29 , for the aforementioned resonance mass range.
\end{abstract}

36th International Conference on High Energy Physics,

July 4-11, 2012

Melbourne, Australia

${ }^{*}$ Speaker. 


\section{Introduction}

The Randall-Sundrum (RS) model [1, 2] was proposed in 1999 in order to address what is known as the hierarchy problem: the apparent discrepancy between the electroweak symmetry breaking scale (Electroweak Scale) and the scale where quantum gravity effects are posited to become strong (Planck scale). Phenomenology of the RS model leads to the appearance of a series of Kaluza-Klein states for the gravitational field quantum, the graviton. Those states appear as massive spin-2 resonances; the first such resonance is known as simply the RS graviton, $\mathrm{G}_{K K}$. Phenomenologically, the model has two parameters: the graviton mass $\left(M_{G}\right)$ and the ratio of the 5-dimensional curvature to the reduced Planck mass $\left(k / M_{P l}\right)$ which acts as the coupling constant of the model.

The process chosen for this study was $\mathrm{pp} \rightarrow \mathrm{G}_{K K} \rightarrow \mathrm{ZZ} \rightarrow \mathrm{q} \overline{\mathrm{q}} v \bar{v}$. The experimental signature of events originating from that reaction is the presence of a single, high-energy, massive hadronic jet. Prospective signal events would also present a large unbalance of energy in the plane transverse to the colliding protons direction, due to the presence of the undetected neutrinos in the final state. A particular feature of this study was the advent of the boosted regime: due to the high boost of the $\mathrm{Z}$ bosons from the graviton decay, the hadronization products from the $\mathrm{Z}$ decay quarks are reconstructed as a single jet in the detector, with invariant mass close to the $\mathrm{Z}$ boson mass. That feature is explored in the selection of prospective signal events in the analysis and rejection of Standard Model (SM) background.

\section{Experimental Setup and Methods}

This analysis uses data corresponding to $4.7 \mathrm{fb}^{-1}$ of proton-proton collisions at a centre-ofmass energy of $7 \mathrm{TeV}$ collected by the Compact Muon Solenoid (CMS) detector during 2011. The central feature of the CMS apparatus is a superconducting solenoid of $6 \mathrm{~m}$ internal diameter, providing a magnetic field of $3.8 \mathrm{~T}$. Within the superconducting solenoid volume are a silicon pixel and strip tracker, a lead tungstate crystal electromagnetic calorimeter (ECAL), and a brass/scintillator hadron calorimeter (HCAL). Muons are measured in gas-ionization detectors embedded in the steel return yoke. Extensive forward calorimetry complements the coverage provided by the barrel and endcap detectors. A more detailed description can be found in Ref. [3]. Event generators PYTHIA [4] and MADGRAPH [5] are used for simulation of both the new physics signal process and the simulated SM background, in order to guide the studies. The GEANT framework [6] is used for the detailed simulation of the CMS detector.

\section{Event Selection}

Candidate events are selected from the collision data through a trigger requiring the presence of at least one calorimetric jet and a significant transverse imbalance of calorimetric energy. To select events with a high probability of originating from real collisions, candidate events are required to have at least one primary vertex of good quality, where the vertex is reconstructed within a 24 $\mathrm{cm}$ window along the beam axis, with a transverse distance from the beam spot of less than $2 \mathrm{~cm}$. Electrons are reconstructed in CMS through the matching of an energy deposit in the electromagnetic calorimeter to a track reconstructed in the tracking system. Muons are reconstructed through 
the matching of signals in the muon spectrometer to a track. Other particle candidates are reconstructed in CMS with the particle flow algorithm, which reconstructs and identifies single particles by combining information from all sub-detector systems. Jets of particles are clustered using the anti- $k_{\mathrm{T}}$ [7] algorithm with a distance parameter $\mathrm{R}=0.7$. The missing transverse energy $E_{\mathrm{T}}^{\text {miss }}$ is defined as the modulus of the negative vector sum of all particles.

Further requirements are imposed on events to select those with a high probability of originating from a prospective signal process. The main requirements are the presence of a jet with transverse momentum $p_{\mathrm{T}}>300 \mathrm{GeV}$ and absolute value of the pseudorapidity $|\eta|<2.4$, the presence of missing transverse energy $E_{\mathrm{T}}^{\text {miss }}>300 \mathrm{GeV}$, and the absence of isolated electrons, muons or tracks. In order to reduce the contamination from multijet background processes, events with more than two jets with $p_{\mathrm{T}}$ above $30 \mathrm{GeV}$ are discarded. Events where there are exactly two jets above $30 \mathrm{GeV}$ are retained, if the azimuthal distance between the two jets is smaller than 2.8 , in order to increase selection efficiency for signal while reducing the number of QCD dijet background events. The sample of candidate signal events is defined as the set of events which pass this selection and also two extra requirements: the invariant mass of the leading jet, $M_{j}$, is larger than $70 \mathrm{GeV}$, and the jet $-E_{\mathrm{T}}^{\text {miss }}$ transverse mass is larger than $900 \mathrm{GeV}$. Events which meet all other requirements but those two are retained to form sideband regions which will be used for a data driven background estimation. Figures $1 \mathrm{a}$ and $1 \mathrm{~b}$ show the two-dimensional distributions for the simulated Standard Model backgrounds, for a simulated signal sample with $M_{G}=1250 \mathrm{GeV}$.

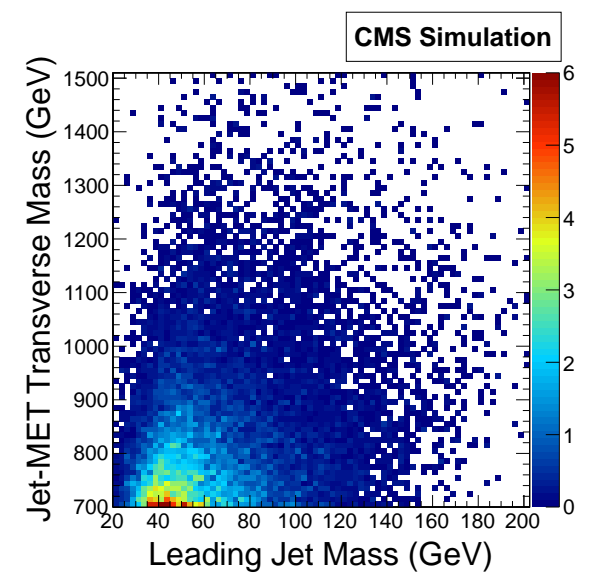

(a)

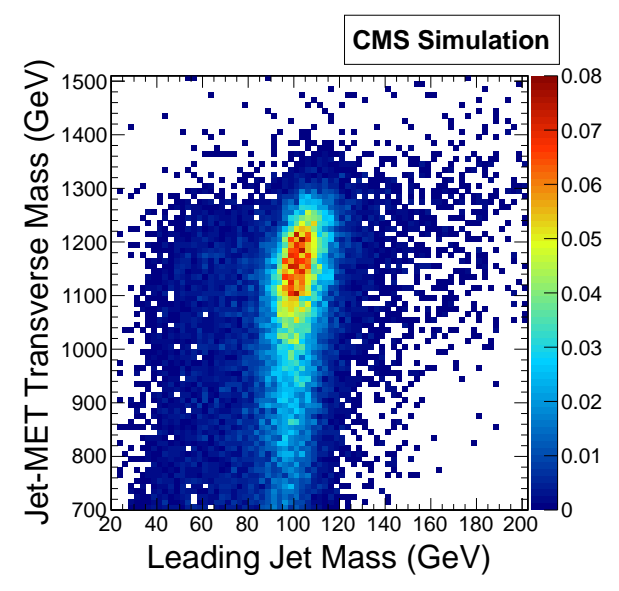

(b)

Figure 1: Joint distributions of leading jet mass and jet- $E_{\mathrm{T}}^{\mathrm{miss}}$ transverse mass, for (a) Standard Model simulated samples (b) RS sample with $M_{G}=1250 \mathrm{GeV}$ and $k / M_{P l}=0.05$.

\section{Background Determination}

The composition of the remaining Standard Model backgrounds in the selected events sample is estimated from simulation to be dominated by $\mathrm{Z} \rightarrow v \bar{v}+$ jet and $\mathrm{W} \rightarrow \ell v+$ jet processes, with the lepton failing to be reconstructed in the latter. In order to estimate the magnitude of the remaining backgrounds after the selection procedure, sideband regions in are defined according to thresholds in the variables $M_{j}$ and $M_{T}$ and events are classified into four categories: 
- Category A: $M_{j}>70 \mathrm{GeV}$ and $M_{T}>900 \mathrm{GeV}$.

- Category B: $20 \mathrm{GeV}<M_{j}<70 \mathrm{GeV}$ and $M_{T}>900 \mathrm{GeV}$

- Category C: $20 \mathrm{GeV}<M_{j}<70 \mathrm{GeV}$ and $700 \mathrm{GeV}<M_{T}<900 \mathrm{GeV}$

- Category D: $M_{j}>70 \mathrm{GeV}$ and $700 \mathrm{GeV}<M_{T}<900 \mathrm{GeV}$

Category A corresponds to the signal region, while categories B, C and D correspond to the sideband regions. The event yields in each category are denoted by $N_{A}, N_{B}, N_{C}, N_{D}$, respectively. Table 1 shows the event yields in each category for simulated SM samples, for the observed data, and the ratio data/simulation for the four categories.

Table 1: Event yields in the four boxes for simulated SM samples, for data, and the ratio data/simulation in the four regions. The quoted uncertainties take into account the statistical error of the simulated samples.

\begin{tabular}{cccc}
\hline Yield & Run2011 Data & SM Prediction & Ratio \\
\hline$N_{A}$ & 138 & $131 \pm 3$ & $1.05 \pm 0.02$ \\
$N_{B}$ & 125 & $125 \pm 3$ & $1.00 \pm 0.03$ \\
$N_{C}$ & 542 & $579 \pm 7$ & $0.94 \pm 0.01$ \\
$N_{D}$ & 283 & $259 \pm 5$ & $1.09 \pm 0.02$ \\
\hline
\end{tabular}

In general, the estimated background $B_{\text {est }}$ in Category $\mathrm{A}$ is given by the expression:

$$
B_{\text {est }}=N_{D} \cdot \frac{N_{B}}{N_{C}} \cdot \frac{1}{\rho}
$$

where $\rho$ is a correction factor to account for the correlation in between the variables $M_{j}$ and $M_{T}$. In this analysis, $\rho$ is estimated directly from the SM simulated samples, by inverting Eq. 4.1 and substituting the simulated event yields:

$$
\rho=\frac{N_{D}}{N_{A}} \cdot \frac{N_{B}}{N_{C}} \quad \text { (yields from simulation). }
$$

Substituting the values from Tab. 1 in Eq. 4.2, we arrive to a value of $\rho=0.42 \pm 0.02$. It is found that, although the value of $\rho$ depends on the definition of the sideband regions, the changes on the estimated background $B_{\text {est }}$ are stable within 5\%. The final value to be used in the mean estimated background from $B_{\text {est }}=153 \pm 29$ events, which is in agreement with the event yield in Category $\mathrm{A}, N_{A}=138$ events. Figures $2 \mathrm{a}$ and $2 \mathrm{~b}$ show the distributions of leading jet mass and jet $-E_{\mathrm{T}}^{\text {miss }}$ transverse mass for Category A events, for simulated backgrounds, simulated signal and observed.

\section{Limit Setting}

In the absence of evidence for an excess of events above that predicted by the Standard Model background, we proceed to set limits on the cross-section of new physics process which would 


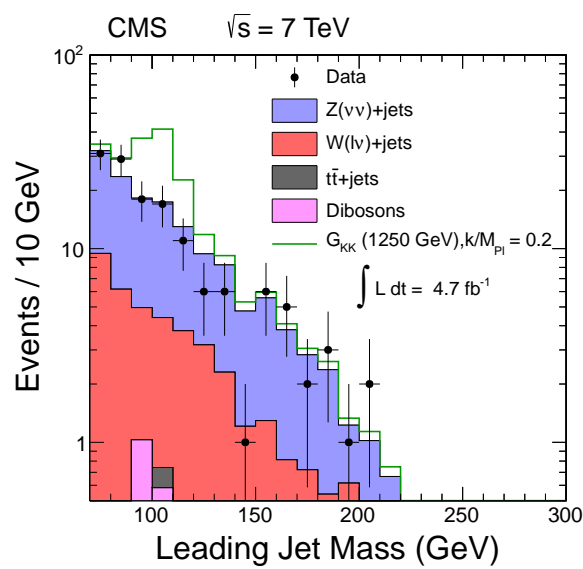

(a)

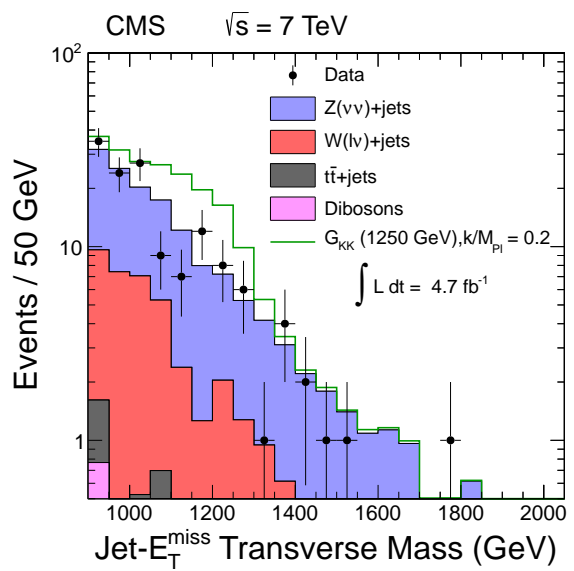

(b)

Figure 2: Comparison between simulated backgrounds in Category A, corrected with the usage of the $\rho$ parameter, and Run2011 data for (a) leading jet mass and (b) jet- $E_{\mathrm{T}}^{\text {miss }}$ transverse mass. Also shown is a prospective $G_{K K}$ signal with $M_{G}=1250 \mathrm{GeV}, k / M_{P l}=0.2$.

entail such an excess. A likelihood for the event yields in the four different regions is setup, with a parameter of interest $\sigma$ representing the cross-section of the prospective new physics process, and nuisance parameters $\mathscr{L}, \varepsilon, n_{i}$ and $\rho$ representing the luminosity, signal selection efficiency, mean expected yields in the sideband regions and the correlation correction parameter respectively. The presence of nuisance parameters is treated with the Profile Likelihood Ratio method. The upper limit on the value of the new physics cross-section times branching ratio $\sigma \times B$ is set using the $\mathrm{CL}_{\mathrm{s}}$ method as implemented in the ROOSTATs framework [8]. Figure 3 shows the upper limits on the cross-section as a function of the assumed resonance mass. The cross-section $95 \%$ confidence upper limits are found to be in the range $[0.047,0.021] \mathrm{pb}$ for the mass of the resonance ranging between 1000 and $1500 \mathrm{GeV}$. This translates to $95 \%$ confidence upper limits on the coupling parameter $k / M_{P l}$ ranging from 0.11 to 0.29 , for the same resonance mass range.

\section{Summary}

A search for a new physics heavy resonance decaying through the reaction $\mathrm{G}_{K K} \rightarrow \mathrm{ZZ} \rightarrow$ $\mathrm{q} \overline{\mathrm{q}} \nu \bar{v}$, was performed in the $4.7 \mathrm{fb}^{-1}$ collected by CMS in 2011. The Randall-Sundrum model was chosen as a benchmark model to study this signature. A set of requirements was applied to the collected data that maximises the amount of prospective signal events selected, while rejecting as much as possible Standard Model background events. A data-driven method was used to estimate the remaining background and, in the absence of any signal above such background, exclusion limits on the cross-section times branching ratio of new physics processes with that signature were set. We conclude that, for a resonance mass in the range $[1000,1500] \mathrm{GeV}, \sigma \times B<[0.047$, $0.021] \mathrm{pb}$ at $95 \%$ confidence level. Interpreting those limits in terms of the Randall-Sundrum model, we conclude that the the existence of the $G_{K K}$ is excluded, at $95 \%$ confidence level, for $k / M_{P l}$ above [0.11, 0.29] in the aforementioned mass range. The Physics Analysis Summary for this analysis can be found in [9]. 


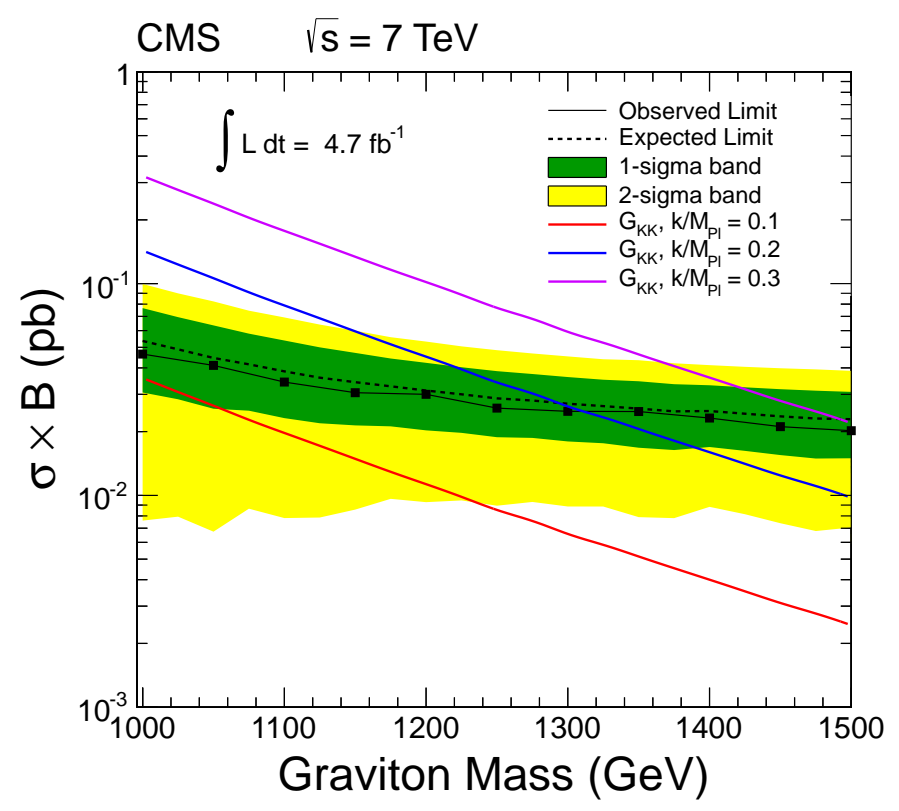

Figure 3: 95\% upper limits on the cross-section times branching ratio for a resonance decaying in $\mathrm{ZZ} \rightarrow$ massive jet $+E_{\mathrm{T}}^{\mathrm{miss}}$. Also shown are the theoretical cross-sections, at leading order, for the production of $\mathrm{G}_{K K}$ with coupling parameters $k / M_{P l}=0.1,0.2$ and 0.3 .

\section{References}

[1] L. Randall and R. Sundrum, "Large Mass Hierarchy from a Small Extra Dimension”, Phys.Rev.Lett. 83 (1999) 3370-3373, doi: 10.1103/PhysRevLett.83.3370, arXiv: hep-ph/ 9905221.

[2] L. Randall and R. Sundrum, “An Alternative to Compactification”, Phys.Rev.Lett. 83 (1999) 4690-4693, doi: 10.1103/PhysRevLett.83.4690, arXiv: hep-th/9906064.

[3] CMS Collaboration, "The CMS experiment at the CERN LHC", JINST 3 (2008) S08004, doi: 10.1088/1748-0221/3/08/S08004.

[4] T. Sjostrand, S. Mrenna, and P. Z. Skands, "PYTHIA 6.4 Physics and Manual", JHEP 0605 (2006) 026, doi: 10.1088/1126-6708/2006/05/026, arXiv:hep-ph/0603175.

[5] M. Herquet and F. Maltoni, "MadGraph/MadEvent : A multipurpose event generator", Nucl.Phys.Proc.Suppl. 179-180 (2008) 211-217, doi: 10.1016/j.nuclphysbps.2008.07.026.

[6] GEANT4 Collaboration, "GEANT4: A Simulation toolkit”, Nucl. Instrum. Meth. A506 (2003) 250-303, doi: 10.1016/S0168-9002(03)01368-8.

[7] M. Cacciari, G. P. Salam, and G. Soyez, "The anti- $k_{t}$ jet clustering algorithm”, JHEP 0804 (2008) 063, doi: 10.1088/1126-6708/2008/04/063, arXiv:0802.1189.

[8] L. Moneta, K. Cranmer, G. Schott et al., "The RooStats Project", Proceedings of the 13th International Workshop on Advanced Computing and Analysis Techniques in Physics Research (2010). http://arxiv.org/pdf/1009.1003v2

[9] CMS Collaboration, "Search for Randall-Sundrum Gravitons Decaying into a Jet plus Missing ET at CMS”, CMS Physics Analysis Summary EXO-11-061 (2012).

http: / / cdsweb. cern. ch/record/1426654. 